\title{
Evolution and Market Share of Wireless Community Networks
}

\author{
Mohammad Hossein Manshaei, Peter Marbach, and Jean-Pierre Hubaux
}

\begin{abstract}
Wireless community networks that operate in the unlicensed spectrum have emerged as a low-cost alternative for providing high-speed wireless data services. By using WiFi access points that are provided and managed by community members, wireless community networks can offer high-speed data services at a much lower cost compared with traditional licensed band operators. However, depending on the number of members, the quality-of-service in terms of coverage and data rates provided by wireless community networks can be significantly lower compared with traditional licensed band operators. As a result, it is not clear whether wireless community networks will be able to capture a significant share of the market for wireless data services. In this paper we use a game-theoretic approach to model and study the evolution, and potential market share, of wireless community networks. We also study whether it is profitable for a licensed band provider to complement its licensed band data service with a low-cost service based on a wireless community network.
\end{abstract}

\section{INTRODUCTION}

Over the last few years we have witnessed a rapidly increasing demand for high-speed wireless data services. Traditionally, these services have been offered by cellular wireless service providers that operate in the licensed-band of the radio spectrum. Cellular wireless providers can guarantee a high-quality service and good coverage. However, providing this service requires substantial investments, both for deploying and maintaining the network infrastructure, and for licensing the wireless spectrum. As a result, subscription fees to high-speed wireless data services provided by a licensedband cellular provider tend to be high.

As an alternative to licensed-band operators, wireless community networks have emerged as a low-cost approach to provide high-speed wireless data service. Wireless community networks operate in the unlicensed-band and use WiFi access points that are operated and maintained by community members to provide their data service. Some wireless community networks are currently operated by FON, a worldwide WiFi community network funded by Google and Skype [2], as well as by Free [3]. Recently, some researchers have also shown their interests in this subject [8][1].

Although wireless community networks offer data services at a much lower cost compared with traditional licensed-band operators, the quality-of-service they provide might also be much lower than that offered by licensed-band operators. For example, the coverage of a wireless community network

M. H. Manshaei and J.-P. Hubaux are with the Laboratory for Computer Communications and Application (LCA), the School of Computer \& Communication Science, EPFL, 1015 Lausanne, Switzerland. \{hossein.manshaei, jean-pierre.hubaux\}@epfl.ch

P. Marbach is with the Department of Computer Science, University of Toronto, Toronto, Ontario, M5S 3G4 Canada. marbach@cs.toronto.edu depends on the number of members it is able to attract. As a result it is not clear whether wireless community networks can indeed emerge as a viable alternative to cellular wireless network providers, i.e. whether wireless community networks will be able to attract a large enough number of subscribers that indeed allows them to compete with traditional licensed-band operators.

In this paper we study wireless community networks using a game-theoretic framework. We start out by analyzing the evolution of wireless community networks. Our analysis suggests that wireless communities exhibit an interesting threshold behavior: If the subscription price that the network charges its users exceeds a certain threshold (which depends on the initial community size), then the network will not be able to grow, and will eventually lose all its members. Whereas, if the price is below this threshold price, then the community will be able to grow.

Next, we consider the competition between a licensedband wireless operator and a wireless community network. For this case, our results suggest that the wireless community networks are more competitive in areas with a high-density WiFi access points. The intuition behind this result is that a high density of WiFi access points is needed for the wireless community network to provide sufficiently good coverage to its subscribers. Finally, we study whether it is profitable for a licensed-band provider to offer a high-speed data service based on a wireless community networks as a low-cost alternative to price-sensitive users. Our results suggest that this is not the case, i.e. in order to maximize its profit, a licensed-band provider should only offer high-speed data service through an appropriately priced licensed-band service. The intuition behind this result is that a unlicensed service based on a wireless community network will add market share, but also erodes profits as some users will switch from the more expensive licensed-band service to the low-cost service offered by the community network.

The paper is organized as follow. In Section II we discuss our economic model for wireless service providers and we also address the scenario that we consider in this paper for mobile subscribers. Section III, IV, and V present our analytical models for users' payoff and wireless providers. In Section VI, we address our main results and contributions. We analyze the revenue of traditional licensed band operators in Section VII. We then evaluate the dynamics of wireless community in Section VIII. In Section IX and X, we analyze using a game-theoretic approach the competition and cooperation between these operators. Finally we conclude with Section XI. 


\section{Problem Formulation}

We consider the situation where users can obtain highspeed data service either through a licensed-band cellular wireless provider, or an unlicensed-band wireless community network. In the following, we will refer to the traditional licensed band operator as an LBO (for licensed-based operator), and the wireless community network as SCO (for social community operator). The LBO provides the service through a fixed infrastructure, thus guaranteeing both good coverage and high-data rates. The SCO, on the contrary, relies on WiFi access points owned by community members to provide the service. To obtain good coverage, the community network has to be able to attract a large number of subscribers.

Users decide which operator to join, based on both the subscription fee that the operator charges and the qualityof-service the operator provides. We denote with $P_{\ell}$ the subscription fee charged by the LBO, and with $P_{S}$ the fee charge by the SCO. Similarly, we denote with $Q_{\ell}$ the qualityof-service provided by the LBO, and by $Q_{s}$ the quality-ofservice of the SCO. Without loss of generality we assume that $Q_{\ell}=1$. In this case, $Q_{s}$ models the quality-of-service provided by the wireless community networks relative to the one provided by the the licensed-band operator. We describe the exact model for the quality-of-service $Q_{s}$ in Section III.

We assume that operators will decide on the subscription price in order to maximize their profit. For our analysis, we consider the following two pricing models.

Definition 1: Under the static pricing models, operators have a fixed subscription fee that does not change over time.

Definition 2: Under the dynamic pricing model, operators can change their subscription fees at regular intervals such as every month, or every year.

For these pricing models, we study the following questions.

1) What are the subscription fees that operators should charge to maximize their profit?

2) If a licensed-band operator and a wireless community network compete with each other in a given area, what is the equilibrium market share of each operator, and what are the subscription fees charged at market equilibrium?

3) Should a licensed-band operator complement its service with a low-cost service based on a wireless community network? If so, what subscription fee should it charge for each service, and what is the market share that each service will obtain?

We study the above questions using a game-theoretic model as described in Sections III-V. As part of the model, we have to specify precisely (1) the quality-of-service provided by the wireless community network (see Section III), (2) the profit an operator obtains (see Section IV), and (3) how users decide to which operator to subscribe (see Section V).

For our analysis, we assume that there are a total of $N$ users that potentially subscribe to a data service. At discretetime intervals $t=0,1,2 \ldots$, users can decide to which operator they want to subscribe. We denote with $n_{\ell}(t)$ the fraction of users that subscribe at time $t$ to the licensed-band operator and with $n_{s}(t)$ the fraction of users that subscribe at time $t$ to the wireless community network. Note that $n_{\ell}(t)=1$ states that at time $t$ all users subscribe to the licensed-band operator. Users are allowed to decide to not subscribe to any operator and we can have that $n_{s}(t)+n_{\ell}(t)<1$.

\section{QuAlity-OF-SERVICE PROVIDEd BY THE WIRELESS COMMUNiTy NETWORK}

In our analysis, we assume that the quality-of-service that is provided by the wireless community network depends on the faction of users $n_{s}$ that subscribe to its service. Intuitively, the larger the number of users that join the community network is, the better the coverage and the quality-of-service $Q_{s}$ provided by the network is. However, if the number of users in the community network becomes too large, then the quality-of-service $Q_{s}$ may drop due to interference between $\mathrm{WiFi}$ access points in the community network. To capture this behavior, we consider in our analysis the four models of Fig. 1 to characterize the relation between the fraction of users $n_{s}$ that subscribes to the community network and the quality-of-service $Q_{s}$ of the wireless community network. Model-A of Fig. 1 is the simplest model, and Model-D is the most sophisticated (albeit still very simple).
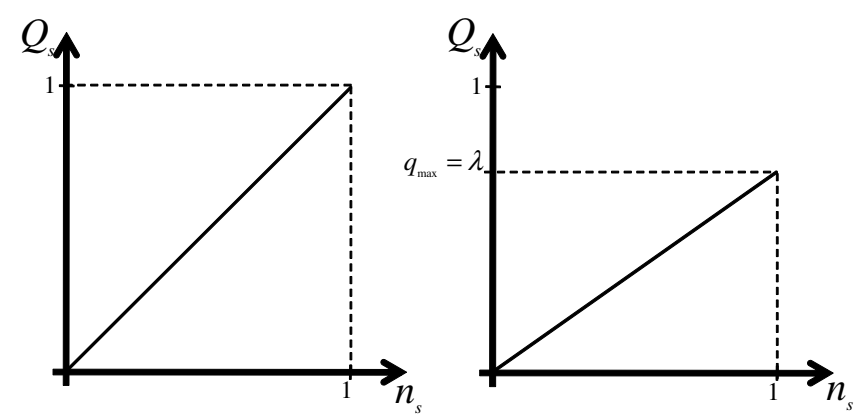

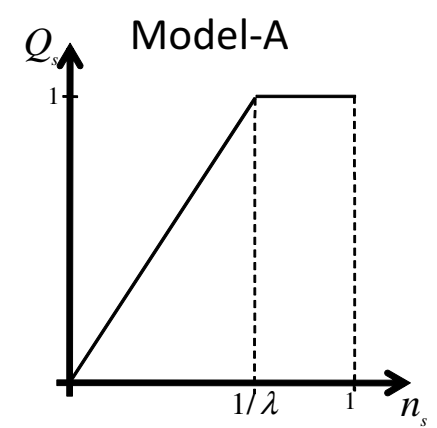

Model-C

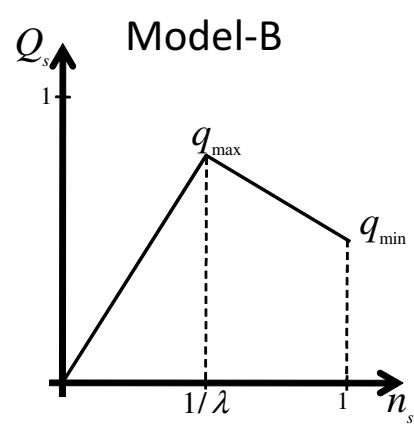

Model-D
Fig. 1. Relation between the quality-of-service $Q_{s}$ provided by the wireless community network and the fraction of users $n_{s}$ that subscribe to the wireless community network.

Model-A assumes a linear relation between the fraction of users $n_{s}$ that subscribe to the wireless community networks and the quality-of-service $Q_{s}$. Furthermore, when $n_{s}=1$, then the quality-of-service of the wireless community network is 
as good as the one provided by the licensed-band operator, i.e. we have $Q_{s}(1)=1$.

Model-B is similar to Model-A, however even when all users subscribe to the wireless community network and $n_{s}=$ 1 , the quality-of-service that the wireless community network can provide is strictly worse than the one of the licensed band operator, i.e we have $Q_{s}(1)=\lambda<1$.

Under Model-C, the wireless community network achieves the same quality-of-service as the one of the licensed band operator, as long as at least a fraction equal to $1 / \lambda$ of the users joins the community networks.

Finally, under Model-D the quality-of-service of the wireless community network deteriorates when more than a fraction $1 / \lambda$ of the users joins the community networks. This degradation in the quality-of-service models the situation where congestion among users, and interference between WiFi access points, in the community network reduces the achievable data rates.

Note that the parameter $\lambda$ in the above model can be interpreted as the density of WiFi access points in a region. The higher the density $\lambda$ is, the lower the fraction of users that have to join the wireless community network in order to provide full coverage (as in Model-C), or create interference (as in Model-D) is.

\section{USER UTILITIES}

Suppose that a given user $v$ subscribes to the licensed-band operator. Then we assume that the total utility (or payoff) that user $v$ obtains is given by

$$
u_{v}^{\ell}=a_{v}-P_{\ell}
$$

where $P_{\ell}$ is the subscription fee (price) of the licensed band operator and $a_{v}$ models the utility that user $v$ obtains under the quality-of-service $Q_{\ell}=1$ of the licensed band operator.

Similarly, the total payoff that user $v$ obtains when subscribing to the wireless community network is given by

$$
u_{v}^{s}=a_{v} Q_{s}-P_{s}
$$

where $Q_{s}$ is the quality-of-service provided by the wireless community network and $P_{s}$ is its subscription fee.

Note that we can interpret $a_{v}$ as a user type parameter that characterizes the sensitivity of user $v$ towards qualityof-service, which determines the willingness of a user to subscribe to a given service provider. The sensitivity towards quality-of-service could obey different distributions over the set of users. In our analysis, we use a probability density function to characterize the fraction of users with a given user type parameter $a_{v}$ as illustrated in Fig. 2.

A given user $v$ only subscribes to a data service if the resulting payoff is positive, i.e. if

$$
\max \left\{a_{v}-P_{\ell}, a_{v} Q_{s}-P_{s}\right\}>0 .
$$

If both payoffs for the licensed band operator and the wireless community networks are positive, then user $v$ will subscribe to the service that provides the higher payoff.
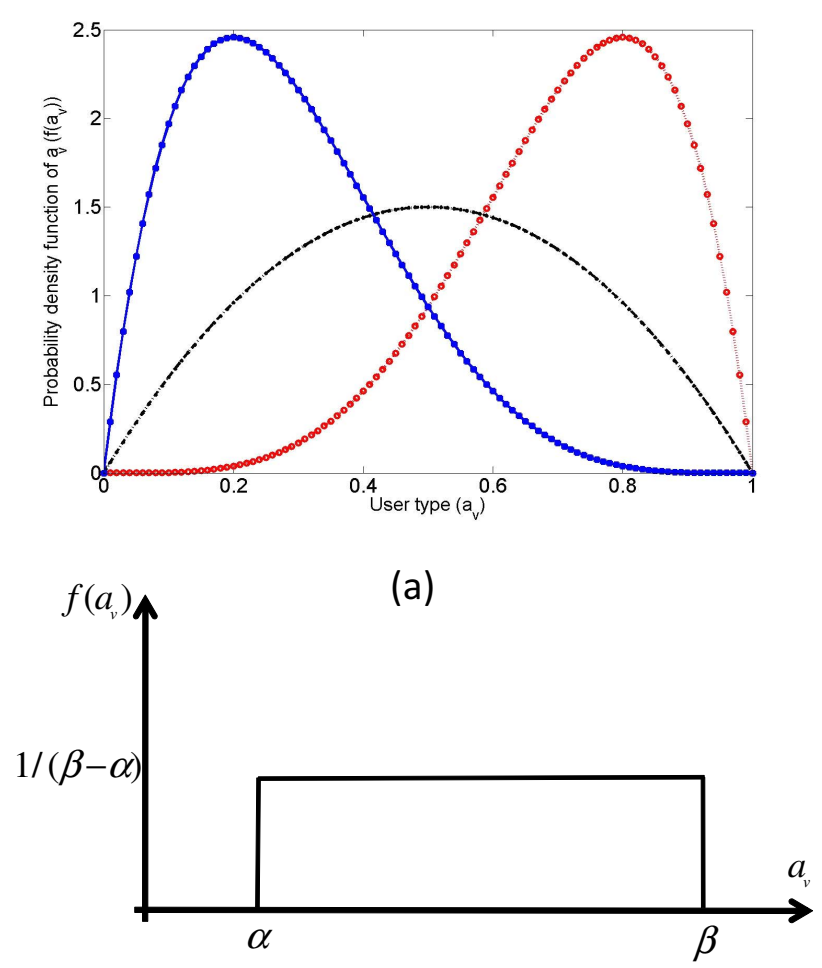

(b)

Fig. 2. Different probability density functions for the user type $a_{v}$. Graph (a) is the beta distribution with different parameters. For our analysis, we will mostly use the uniform distribution over an interval $[\alpha, \beta]$ as given in graph (b).

\section{Payoff of the Wireless Service Providers}

We denote with $u_{\ell}$ and $u_{s}$, the payoffs of the licensed-band operator and wireless community network, respectively.

The payoff $u_{\ell}$ of the licensed-band operator is given by

$$
u_{\ell}=N \cdot n_{\ell} \cdot P_{\ell}-c_{\ell},
$$

where $N$ is the total number of users, $n_{\ell}$ is the fraction of users that subscribe at time $t$ to the licensed-band operator, and $c_{\ell}$ models the operation costs of the licensed-band service provider. The $\cos t c_{\ell}$ is the fixed cost used to maintain and operate the infrastructure.

Similarly, the payoff of the wireless community network operator is given by

$$
u_{s}=N \cdot n_{s} \cdot P_{s}-c_{s} .
$$

Note that the fixed $\cos t c_{s}$ to operate the wireless community network is much smaller than the cost $c_{\ell}$ for the licensedband operator as the infrastructure to provide wireless access is provided by the community members.

\section{MAin Results}

In this section we provide an overview of the main results that we obtained under the above models. We evaluate the model in two scenarios. Throughout this paper, we assume 
that $a_{v}$ is uniformly distributed in $[\alpha, \beta]$ (i.e. $\left.f\left(a_{v}\right)=\frac{1}{\beta-\alpha}\right)$, where $\alpha \geq 0$ and this distribution is known to the operators.

First we consider a monopoly scenario where there exists only one operator in the service area. We analyze the revenue of LBO and concluded that its revenue depends on the distribution of user types. We also calculate the optimal pricing strategy for LBO that maximizes its revenue, i.e. $P_{\ell}^{o p t}=\max \{\alpha, \beta / 2\}$ as shown in Section VII.

The dynamics of SCO in monopoly scenario depends not only on the distribution of user types, but also on its initial quality-of-service $Q_{s}[0]$ and the relation between the number of subscribers and provided quality-of-service. Hence, we use the four types of functions that can model the relation between the quality-of-service of the SCO and the number of its subscribers as shown in Fig. 1. As discussed in Section III, our quality-of-service models can also characterize the situations where there is collision between the access points and among subscribers, which reduces the achieved data rate of subscribers. Using these models, we first identify the equilibrium points of SCO quality-of-service where the quality-of-service of the community could potentially converge to them. Considering the dynamics of SCO and its equilibrium points, we then compute the optimal subscription fee with static and dynamic price strategy which maximize the revenue of SCO. We show that for any given distribution of user type and initial quality-of-service, the operator can obtain the optimal price if he knows the relation between its number of subscribers and its quality-of-service in the service area. The calculated optimal prices also depends on the initial quality-of-service and distribution of user types for all quality-of-service models. For example, if the initial quality-of-service is small, then the SCO needs to choose a subscription fee small enough in order to obtain more subscribers and increase its quality-of-service. Whereas, when the initial quality-of-service is large enough, the optimal pricing strategy does not depend on initial quality-of-service (e.g., $P_{S}^{o p t}=\frac{2 \lambda}{9} \frac{\beta^{2}}{\beta-\alpha}$ that depends only on distribution of user types and quality-of-service model).

Second, we consider the co-existence of a LBO and a SCO. We model this scenario with a game-theoretic approach and compute the operators' best responses in term of their subscription fees. We show that under certain conditions there exists a Nash equilibrium for the market share between two operators and we calculate their respective payoffs at this equilibrium. The Nash equilibrium strategy profile depends on distribution of user types and quality-of-service model of wireless community as presented in Section IX. These results could help operators to select their best strategy in competition with other operators in any given service area and for any given distribution of user types.

We finally evaluate the potential benefits of deploying two wireless access services by one operator and we show that under uniform distribution of user types (no matter what $\alpha$ and $\beta$ are) and with quality-of-service Model-A there is no interest for an operator to deploy both services simultaneously. In this case, the optimal pricing strategy of the operator corresponds to the one of LBO.

In summary, using our model we are able to answer the questions that presented in Section II. This means that according to our results the operators can calculate the optimal pricing strategies that maximizes their payoffs as a function of distribution of user types and their qualityof-services. The operators are also able to find the Nash equilibrium for market share if they coexist in the same service area and calculate the corresponding subscription fees. Finally, they are able to examine the potential benefits of deploying both wireless access services at the same time for a given service area and distribution of user type.

\section{PRofit Maximization of the LiCEnsed BAnd OPERATOR}

Consider the situation where the LBO is the only wireless access provider in a given area. For this case, we are interested in determining the optimal price $P_{\ell}^{o p t}$ that the LBO should charge per unit of time in order to maximize its revenue. Note that under a given price $P_{\ell}$, only user for which the payoff $u_{v}^{l}$ given by Equation (3) is non-negative subscribes to the LBO. For any given distribution of user type, the fraction of users $n_{\ell}$ that subscribe to the LBO under price $P_{\ell}$ can be calculated by (recall that $Q_{\ell}=1$ for LBO):

$$
n_{\ell}=\operatorname{Prob}\left\{a_{v}>P_{\ell}\right\}=\int_{P_{\ell}}^{\infty} f\left(a_{v}\right) d a_{v} .
$$

For example, one can prove that the optimal subscription fee for the uniform distribution of user types over $[\alpha, \beta]$ is $P_{\ell}^{o p t}=$ $\max \left\{\alpha, \frac{\beta}{2}\right\}$. The fraction of users $n_{\ell}^{o p t}$ that subscribes to the LBO under the price $P_{\ell}^{o p t}$ is given by $n_{\ell}^{o p t}=\max \left\{1, \frac{1}{2} \frac{\beta}{\beta-\alpha}\right\}$.

The above results show that the optimal price and the number of subscribers of an LBO depend on the distribution of user types.

\section{EVOLUTION OF THE WiRELESS COMMUNity NETWORK}

Next, we assume that the SCO is the only wireless access provider and we study the evolution of its network. Again we assume that user $v$ will subscribe to this operator at time $t$, if and only if $u_{v}^{s}$ is strictly greater than zero for a given quality-of-service $Q_{s}[t-1]$. Similar to the LBO we calculate the fraction of subscribed users at time $t$ by

$$
n_{s}[t]=\operatorname{Prob}\left\{a_{v}>P_{s} / Q_{s}[t-1]\right\}=\int_{P_{s} / Q_{s}[t-1]}^{\infty} f\left(a_{v}\right) d a_{v} .
$$

Note that if the initial quality-of-service is equal to zero (i.e., $Q_{s}[t-1]=0$ ) the wireless community never forms. We denote the difference in terms of quality-of-service between two time steps $t$ and $t-1$, by $\Delta Q_{s}=Q_{s}[t]-Q_{s}[t-1]$, where positive and negative values of $\Delta Q_{s}$ express the improvement and degradation of the provided quality-of-service of SCO at time $t$, respectively. We also define the equilibrium of an SCO as follows:

Definition 3: For given values of $P_{s}$ and $Q_{s}[t-1]$, the SCO is in an equilibrium point $Q_{s}^{e q}$, if $\Delta Q_{s}=0$. 
In the following sections we evaluate the dynamics of SCO under different quality-of-service models, shown in Fig. 1. Recall that $a_{v}$ is uniformly distributed in $[\alpha, \beta]$ (Fig. 2(b)) but the results can be extended to more general cases with different distributions of user types.

\section{A. Evolution of Wireless Community with Model-A}

Using Model-A, we can calculate the number of subscribers and provided quality-of-service by:

$$
Q_{s}[t]=n_{s}[t]=\frac{1}{\beta-\alpha}\left(\beta-\max \left\{\alpha, \frac{P_{s}}{Q_{s}[t-1]}\right\}\right)
$$

Consequently, $\Delta Q_{s}$ can be expressed as follow:

$$
\begin{array}{rc}
\Delta Q_{s}= & Q_{s}[t]-Q_{s}[t-1] \\
& =\frac{-(\beta-\alpha) Q_{s}^{2}[t-1]+\beta \cdot Q_{s}[t-1]-P_{s}}{(\beta-\alpha) Q_{s}[t-1]},
\end{array}
$$

In [4], a thorough study for the dynamics of community operator with Model-A is presented. The community operator has potentially four equilibrium points (i.e., $\left.Q_{s}^{e q}\right)$ with Model-A; $Q_{s}^{e q}=\left\{0, Q_{s, 1}, Q_{s, 2}, 1\right\}$, where $Q_{s, 1,2}=$ $\frac{\beta \pm \sqrt{\beta^{2}-4(\beta-\alpha) P_{s}}}{2(\beta-\alpha)}$.

We also prove that, under different subscription fees and initial quality-of-service, the quality-of-service of the SCO can converge to one of the above equilibrium points. Fig. 3 illustrates the dynamics of SCO for any given initial qualityof-service at time $t-1$ (i.e., $Q_{s}[t-1]$ ) and subscription fee $P_{s}$, when $\beta>2 \alpha$. We also observe that if the price selected by $\mathrm{SCO}$ is such that $Q_{s}[t-1]$ is less than $Q_{s, 1}$, then the SCO can never increase its quality-of-service and consequently, the proportion of subscribers and its revenue. In other words, $\lim _{t \rightarrow \infty} Q_{s}[t]=0$. Note that we also prove that the convergence of SCO is monotonous [4].

(a)

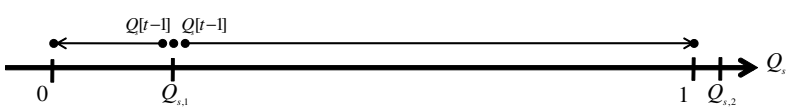

(b)

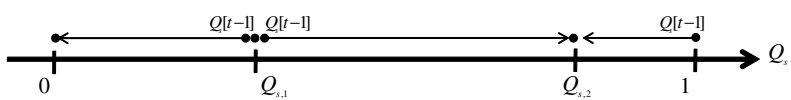

(c)

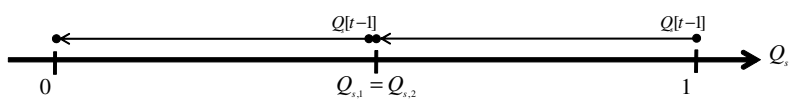

(d)

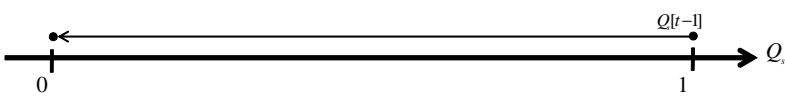

Fig. 3. Dynamics of SCO when $\beta>2 \alpha$ with Model-A: (a) $0<P_{s} \leq \alpha$, (b) $\alpha<P_{s}<\frac{\beta^{2}}{4(\beta-\alpha)}$, (c) $P_{s}=\frac{\beta^{2}}{4(\beta-\alpha)}$, (d) $P_{s}>\frac{\beta^{2}}{4(\beta-\alpha)}$.

Considering the dynamics of SCO under static price, we can also derive the optimal static price that maximizes the payoff as well as market share of the SCO. This optimal price (i.e., $P_{s}^{o p t}$ ) is a function of initial quality-of-service (i.e., $\left.Q_{s}[0]\right)$ and distribution of user type. For example, we show in [4] that if $Q_{s}[0] \leq \frac{\alpha}{\beta-\alpha}$ then $P_{s}^{o p t}=Q_{s}[0] \cdot(\beta-(\beta-\alpha)$. $\left.Q_{s}[0]\right)$. Or the optimal static price is $P_{s}^{o p t}=\alpha$ for $2 \alpha<\beta \leq$ $3 \alpha$ and it is $P_{s}^{o p t}=\frac{2}{9} \frac{\beta^{2}}{(\beta-\alpha)}$ when $\beta>3 \alpha$.
In summary, our results show that the initial quality-ofservice $Q_{s}[0]$, and the distribution of user types determine the range of optimal static prices from which the SCO can select its price. However, when the distribution of user type is wide enough $(\beta>3 \alpha)$ and $Q_{s}[0]>\frac{\alpha}{\beta-\alpha}$, the optimal price does not depend on the initial quality-of-service.

We also evaluate a dynamic pricing strategy of community network in [4]. This means that SCO adjusts its price $P_{S}$ at time $t$ to follow the evolution of its network. The essential difference between static and dynamic pricing is that with dynamic pricing the SCO can maintain a lower price until a desired quality-of-service is reached and then fine-tune the price. The price at each time instance $t$ is a function of quality-of-service at time $t-1$. As $\Delta Q_{s}$ must be strictly positive, the SCO maintains the increase of the quality-ofservice by selecting appropriate dynamic prices $P_{S}[t]$ at time $t$, such that,

$$
P_{s}[t]=-(\beta-\alpha) Q_{s}^{2}[t-1]+\beta Q_{s}[t-1]-\varepsilon
$$

where $\varepsilon$ is a small positive value. Similar to the static price strategy, two main scenarios can be distinguished.

We also calculate the optimal dynamic price strategies and corresponding final quality-of-service. For example, we show that if $\beta>3 \alpha$ the best price and quality-of-service that maximizes the SCO payoff, i.e., $Q_{s}^{\text {opt }}=\frac{2}{3} \frac{\beta}{\beta-\alpha}, P_{s}^{o p t}=\frac{2}{9} \frac{\beta^{2}}{\beta-\alpha}$, and $u_{s}^{o p t}=\frac{4}{27} \frac{\beta^{3}}{(\beta-\alpha)^{2}}-c_{s}$.

\section{B. Evolution of Wireless Community with Model-B and Model-C}

As it is shown in Fig. 1 (b) and (c), we use the following simple relation between $Q_{s}$ and $n_{s}$ to model these situations:

$$
Q_{s}[t]=\min \left\{1, \lambda n_{s}[t]\right\}, \forall \lambda>0
$$

Again we can calculate the quality-of-service $Q_{s}[t]$ of the $\mathrm{SCO}$ at time $t$ as a function of the quality-of-service $Q_{s}[t-1]$ at the previous time step. In particular, we have that

$$
Q_{s}[t]=\min \left\{1, \frac{\lambda}{\beta-\alpha}\left(\beta-\max \left\{\alpha, \frac{P_{s}}{Q_{s}[t-1]}\right\}\right)\right\}
$$

Similar to Model-A, we have studied (a) how the qualityof-service $Q_{s}[t]$ evolves over time under a fixed price $P_{s}$ and (b) what price $P_{s}^{o p t}$ the SCO should charge in order to maximize its revenue. The detailed results are available in [5].

Withe these models, the wireless community has still four equilibrium points $Q_{s}^{e q}=\left\{0, Q_{s, 1}, Q_{s, 2}, 1\right\}$ where $Q_{s, 1,2}=$ $\frac{\beta \lambda \pm \sqrt{\beta^{2} \lambda^{2}-4(\beta-\alpha) P_{s} \lambda}}{2(\beta-\alpha)}$. This shows that the equilibrium points of quality-of-service are the function of $\lambda$ as well.

Our results show that the dynamics of SCO with Model-B and Model-C are quite similar to that of Model-A and depends on initial quality-of-service and selected subscription fee as well as $\lambda$.

For example, if $\beta-\frac{1}{\lambda}(\beta-\alpha)<P_{s}<\frac{\beta^{2} \lambda}{4(\beta-\alpha)}$ and $Q_{s}[0]<$ $Q_{s, 1}$ then we have that $\lim _{t \rightarrow \infty} Q_{s}[t]=0$. Or with the same conditions on price if $Q_{s}[0]>Q_{s, 1}$ then $\lim _{t \rightarrow \infty} Q_{s}[t]=Q_{s, 2}$. 
Similar to Model-A we have also calculated the optimal static prices with which the social community operator can maximize its payoff at one of the defined equilibrium points. For example, if $Q_{s}[0] \leq \lambda \frac{\beta}{\beta-\alpha}-1$, then $P_{s}^{o p t}=Q_{s}[0] \cdot(\beta-$ $\left.\frac{1}{\lambda}(\beta-\alpha) \cdot Q_{s}[0]\right)$ and $n_{s}^{o p t}=1$.

Note that the optimal price $P_{s}^{o p t}$ depends on the initial quality-of-service; the intuition behind this is that when the initial quality-of-service $Q_{s}[0]$ is small, then the SCO needs to choose a subscription fee small enough in order to prevent the quality-of-service $Q_{s}[t]$ to converge to 0 . Whereas, we show that if the initial quality-of-service is large the $P_{s}^{o p t}=$ $\frac{2 \lambda}{9} \frac{\beta^{2}}{(\beta-\alpha)}$ which only depends on $\alpha, \beta$, and $\lambda$, but not on the initial quality-of-service $Q_{s}[0]$. Similar to Model-A, we also calculate the optimal dynamic price strategy for Model-B and Model-C in [5].

\section{Evolution of Wireless Community with Model-D}

As it is shown in Fig. 4, with Model-D the qualityof-service of community increases when the fraction of subscribers is less than $1 / \lambda$ and we obtain the maximum quality-of-service $q_{\max }$ when the fraction of subscribers is equal to $1 / \lambda$. For any given initial quality-of-service $Q_{s}[0]$ we can identify four types of evolution for the wireless community. These scenarios have been shown in Fig. 4 and can be summarized as following:

1) The number of subscribers is increased whereas the quality-of-service is decreased.

2) The number of subscribers and the quality-of-service are increased. The final fraction of subscribers is larger than $1 / \lambda$.

3) The number of subscribers and the quality-of-service are increased. The final number of subscribers is smaller than $1 / \lambda$.

4) The number of subscribers and the quality-of-service are decreased.

We can again calculate the $\Delta Q_{s}$ with Equation (8) and find the conditions on subscription fee with which the above scenarios can take place. Our evaluations show that the operator can select a suitable subscription fee to increase its quality-of-service and revenue and to avoid potential collisions between its access points. Fig. 4 shows the relation between the selected subscription fee and its corresponding evolution scenario. Note that the thresholds $T_{i}, i=1,2,3$ depend on $\lambda, \alpha, \beta, q_{\max }, q_{\min }$, and the initial quality-ofservice.

\section{Market Share of Wireless Community NETWORKS IN A COMPETITIVE MARKET}

In this section, we consider the situation where an LBO and an SCO co-exist in a given service area and compete for mobile users to subscribe to their service. We model this situation as a non-cooperative pricing game where two operators are the players. The operators compete through their subscription price and the the strategy of operator $i$ in the game is given by its price $P_{i}$. We also assume that the relation between the number of subscribers and the provided
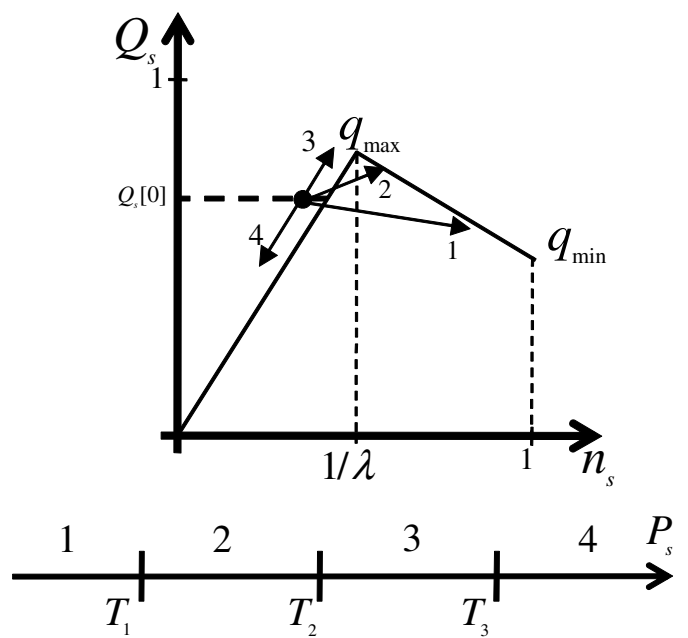

Fig. 4. Relation between provided quality-of-service and number of subscribers for wireless community operators with Model-D and all possible evolution scenarios. For example if the subscription fee is bigger than $T_{3}$, the quality-of-service and number of subscription will be decreased.

quality-of-service of community is modeled by Model-B and Model-C. The evaluation of this game for Model-A is presented in [4].

Again we assume that users make decisions at discrete time steps $t=1,2, \cdots$. Recall the definition of the utility $u_{v}^{i}$ that user $v$ achieves when it subscribes to a provider $i \in\{\ell, s\}$. Given subscription fees $P_{\ell}$ and $P_{s}$, and observing the qualityof-service $Q_{s}[t-1]$ of the SCO at time $t-1$, user $v$ will choose at time $t$ the provider $i$, which leads to the largest utility $u_{v}^{i}$ at time $t$. Of course, user $v$ will only subscribe to this provider if the resulting utility is non-negative; otherwise the user will not subscribe to any provider. Let $n_{\ell}[t]$ and $n_{s}[t]$ the resulting fraction of users that subscribe to the LBO and the SCO, respectively, at time $t$. Considering the payoffs of two operators, the Nash equilibrium [6][7] for the above game is given as follows.

Definition 4: The price pair $\left(P_{\ell}^{*}, P_{s}^{*}\right)$ constitutes a Nash equilibrium if for each operator $i \in\{\ell, s\}$ we have

$$
u_{i}\left(P_{i}^{*}, P_{j}^{*}\right) \geq u_{i}\left(P_{i}, P_{j}^{*}\right), \quad \forall P_{i} \geq 0 .
$$

In other words, at a Nash equilibrium, none of the operators has an incentive to unilaterally change its subscription fee as this would not increase its payoff. If the Nash equilibrium exists for the defined game we can also define the equilibrium quality-of-service and market sharing as follow:

Definition 5: If the price pair $\left(P_{\ell}^{*}, P_{s}^{*}\right)$ constitutes a Nash equilibrium then $Q_{s}^{*}\left(P_{\ell}^{*}, P_{s}^{*}\right)$ is an equilibrium quality-ofservice. Similarly, $n_{\ell}^{*}\left(P_{\ell}^{*}, P_{s}^{*}\right)$ and $n_{s}^{*}\left(P_{\ell}^{*}, P_{s}^{*}\right)$ are the equilibrium market sharing.

In the following we study whether there exists a Nash equilibrium for the above game. To simplify the analysis, we assume that $\alpha=0$.

Theorem 1: Suppose that $\alpha=0$. If $\lambda \in(0,3)$ then there 
exists a unique Nash equilibrium given by

$$
\left(P_{\ell}^{*}, P_{s}^{*}\right)=\left(\frac{\beta}{2} \cdot \frac{1-Q_{s}^{*}}{1-\frac{Q_{s}^{*}}{4}}, \frac{\beta Q_{s}^{*}}{4} \cdot \frac{1-Q_{s}^{*}}{1-\frac{Q_{s}^{*}}{4}}\right)
$$

where $Q_{s}^{*}=2-\sqrt{4-\lambda}$. The fraction of users that subscribe to the SCO at the Nash equilibrium is given by $n_{s}^{*}=\frac{1}{\lambda} Q_{s}^{*}=$ $\frac{1}{2+\sqrt{4-\lambda}}$, and the fraction of users that subscribe to the LBO is given by $n_{\ell}^{*}=\frac{2}{2+\sqrt{4-\lambda}}$.

If $\lambda \geq 3$, then there exists a unique Nash equilibrium $\left(P_{\ell}^{*}=\right.$ $\left.0, P_{s}^{*}=0\right)$ with $Q_{s}\left(P_{\ell}^{*}, P_{s}^{*}\right)=1$. However the fraction of users that subscribe the each operator are not uniquely determined. In particular, any market share $n_{\ell}^{*}$ and $n_{s}^{*}$ such that $n_{s}^{*} \geq 1 / \lambda$ and $n_{\ell}^{*}+n_{s}^{*}=1$ may be realized at a Nash equilibrium.

The above analysis implies that there always exists a unique Nash equilibrium. For $\lambda \in(0,3)$ the market share of each provider is uniquely determined. Furthermore, for $\lambda \in(0,3)$, we have that (a) the market share (i.e. the fraction of users that subscribe to an operator) of both the LBO and SCO increase as $\lambda$ increases and (b) the market share of the LBO is always twice as large as the market share of the SCO.

The subscription fees $\left(P_{\ell}^{*}, P_{s}^{*}\right)$ charged at a Nash equilibrium tend to decrease as $\lambda$ increases. This suggests that the SCO influences the pricing behavior of an LBO, and that the presence of an SCO in the service area with a dense network of WiFi access points might be able to significantly reduce the fees charged for wireless access.

\section{Market Share of Wireless Community NeTwORKS IN A COOPERATIVE MARKET}

The above service providers can also cooperate to obtain more subscribers and provide better service. In other words, the operators cooperator to maximize the total throughput $u_{t}=u_{s}+u_{\ell}$.

Let's consider a uniform distribution of user types with Model-A for the relation between quality-of-service and number of subscribers. Then if both operators have a market share, we can write their payoffs as following:

$$
\begin{gathered}
u_{\ell}=\frac{N}{\beta-\alpha}\left(\beta-\frac{P_{\ell}-P_{s}}{1-Q_{s}}\right)-c_{\ell} \\
u_{s}=\frac{N}{\beta-\alpha}\left(\frac{P_{\ell}-P_{s}}{1-Q_{s}}-\frac{P_{s}}{Q_{s}}\right)-c_{s}
\end{gathered}
$$

We then find the $P_{s}$ and $P_{\ell}$ that maximize $u_{t}$. Using Equation (13) and (14), we obtain a pair $\left(P_{s}^{*}, P_{\ell}^{*}\right)$ that maximizes the total payoff:

$$
\left(P_{s}^{*}, P_{\ell}^{*}\right)=\left(\frac{\beta Q_{s}}{2}, \frac{\beta}{2}\right)
$$

Finally, by introducing $P_{s}^{*}$ and $P_{\ell}^{*}$ in $u_{t}$, we obtain $u_{t}^{*}=u_{\ell}^{*}$. In other words, a global operator (who has both licensed band and community network) will not deploy a community for the uniform distribution of user types as it does not bring any added value. Note that its optimal subscription fee and payoff corresponds to the one of LBO in monopoly scenario presented in Section VII. The evaluation of cooperation for other distribution of user types and quality-of-service models is a part of our future work. This will show how traditional operators can relay on community networks as a complimentary service to increase their benefits.

\section{Conclusions}

In this paper, we have studied how the evolution of social community networks is conditioned by the initial provided quality-of-service, the subscription fee and user sensitivity to the provided quality-of-service. In a monopoly, we have identified the optimal static and dynamic pricing strategies for the wireless community with different models of qualityof-service for wireless community. Our model can help wireless communities to determine when the initial qualityof-service is sufficient and then determine which price to set. The model distinguishes pricing strategies for different distribution of user types and different models of quality-ofservice for wireless communities.

We have also studied the coexistence of the SCO and the LBO. We investigated the existence Nash Equilibrium. We have computed the prices that the operators should use to be at the identified Nash Equilibrium. We have also analyzed the cooperation between the operators using a game-theoretic approach. The results that we obtained are intuitive, suggesting that the simple model that we used is able to capture the main features of the competition or cooperation between a LBO and a SCO.

\section{REFERENCES}

[1] E. C. Efstathiou, P. A. Frangoudis, and G. C. Polyzos. Stimulating participation in wireless community networks. In INFOCOM, 2006.

[2] FON. Fon wireless access provider.

[3] Free ISP. Community of free subscribers: http://www.free.fr.

[4] M. H. Manshaei, J. Freudiger, M. Felegyhazi, P. Marbach, and J.P. Hubaux. On Wireless Social Community Networks. In IEEE INFOCOM, 2008.

[5] M. H. Manshaei, J. Freudiger, M. Felegyhazi, P. Marbach, and J.P. Hubaux. Wireless social community networks: a game-theoretic analysis. In International Zurich Seminar on Communications. IEEE, 2008.

[6] J. Nash. Non-cooperative games. The Annals of Mathematics, 54(2):286-295, 1951.

[7] M. J. Osborne and A. Rubinstein. A Course in Game Theory. The MIT Press, Cambridge, MA, 1994.

[8] A. Zemlianov and G. de Venciana. Cooperation and decision-making in a wireless multi-provider setting. In IEEE INFOCOM. IEEE, 2005. 